\title{
A case report of moderate COVID-19 with an extremely long-term viral shedding period in China
}

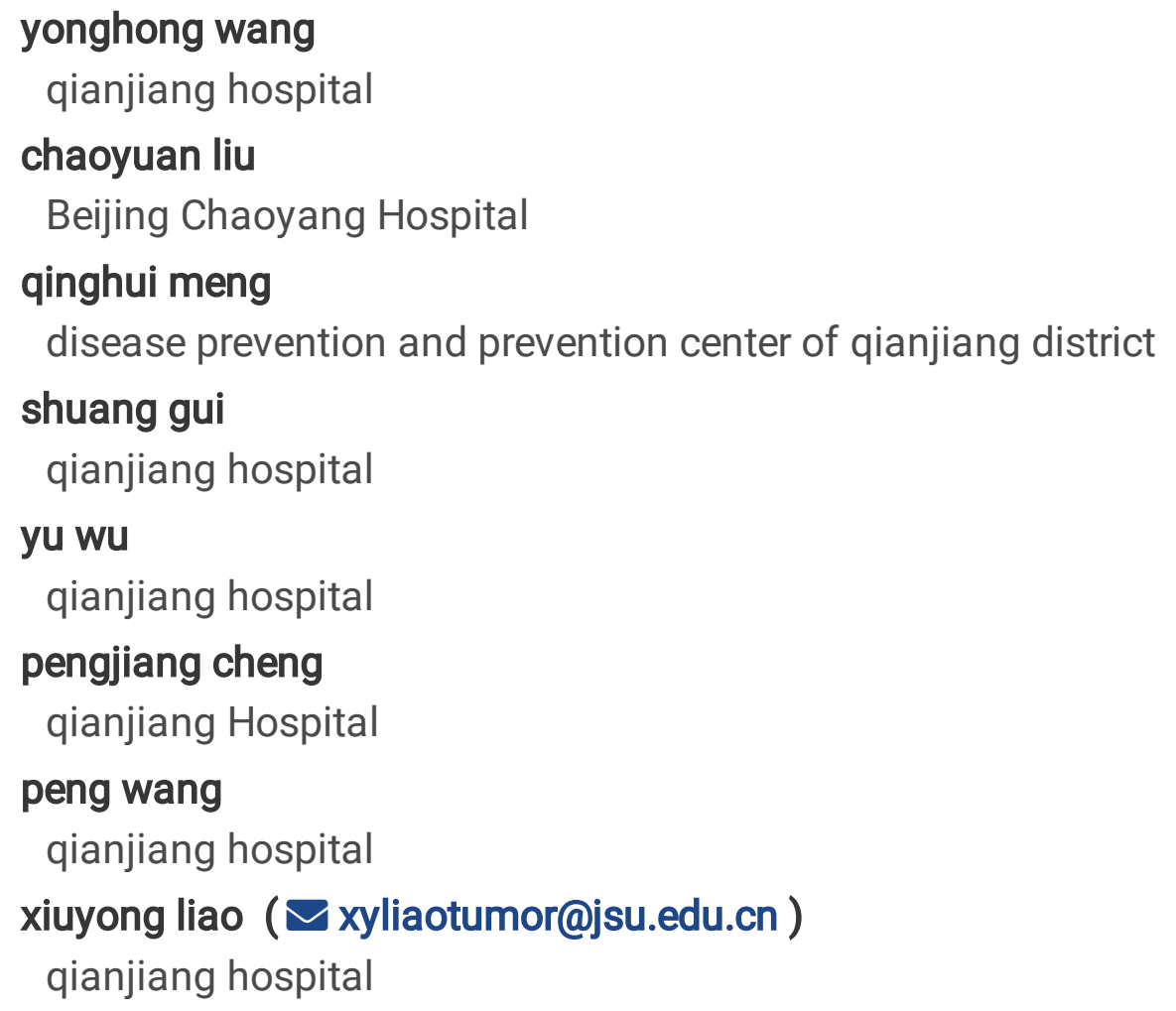

\section{Case Report}

Keywords: COVID-19, IgG antibody, viral shedding, RT-PCR, CD4+ count, case report

Posted Date: August 19th, 2020

DOI: https://doi.org/10.21203/rs.3.rs-59700/v1

License: (c) (1) This work is licensed under a Creative Commons Attribution 4.0 International License. Read Full License 


\section{Abstract}

\section{Background}

An ongoing outbreak of novel coronavirus disease 2019 (COVID-19) from Wuhan, China, is currently recognized as a global public health emergency, which has subsequently spread to the rest of China and other countries. The WHO raised the COVID-19 alert to the highest level. The virus is a new highly contagious via human-to-human transmission. The median duration of viral shedding is 20.0 days. We report that the longest duration of viral shedding was 32.0 days from illness onset in a patient with moderate COVID-19 admitted to QianJiang Central Hospital.

\section{Case presentation}

A 37-year-old male sought medical advice while suffering from fever, dry cough, fatigue, dizziness, runny nose and diarrhoea. Five days before the visit, he had a history of travel from affected geographic areas. The patient had a positive RT-PCR test, and chest CT images showed multiple nodules and mixed groundglass opacification with consolidation in both lungs. Laboratory findings showed that his lymphocyte and $\mathrm{CD} 4^{+}$counts were below the normal range. The patient was given antiviral treatment, including arbidol, lopinavir, IFN- $a$, and traditional Chinese medicine, and other necessary support care. All clinical symptoms and CT imaging manifestation abnormalities resolved during the course of therapy.

\section{Conclusion}

Although the positive RT-PCR tests were verified in consecutive upper respiratory specimens, the clinical symptoms, CT imaging findings, CD4 + lymphocyte counts, and IgG antibody levels had obviously improved. Positive tests may be detecting pieces of inactive viruses, which would not be transmissible in individual cases.

\section{Background}

Since the outbreak of coronavirus disease 2019 (COVID-19, previously known as SARS-CoV-2), which was first reported from Wuhan, China, on 31 December 2019, the causative pathogen has rapidly spread throughout China and around the world in a relatively short period of time. This virus is a positive-sense single-stranded RNA virus belonging to the family Coronaviridae, which is distributed broadly among humans, other mammals, and birds. Most coronaviruses cause a range of mild upper respiratory infections, inducing symptoms of fever, fatigue, and dry cough; some patients present with nasal congestion, runny nose, diarrhoea and shortness of breath[1], but sometimes they exhibit severe acute respiratory syndrome (SARS), coagulation disorders and metabolic acidosis[2].

As of March 7, 2020, the cumulative incidence in mainland China, Hong Kong, and Macau is 80735 cases, of which 67592 cases were diagnosed in Hubei. Additionally, 3045 deaths have been linked to the 
outbreak, and 19903 cases have been reported in South Korea, Iran, Italy, France, Japan, the United States and other countries[3].

China health authorities posted the full COVID-19 genome sequence on January 10, 2020, and the Chinese Center for Disease Control and Prevention (CDC) approved a real-time reverse transcriptasepolymerase chain reaction(RT-PCR) test that can diagnose COVID-19 clinical respiratory specimens[4], including upper (nasopharyngeal and throat swabs) and lower (sputum and bronchial lavage fluid) respiratory tract specimens, anal swabs and faecal samples. Hence, RT-PCR is widely deployed in diagnosis of COVID-19. According to the Chinese management guideline for COVID-19 (standard version 6)[5], negative RT-PCR results from at least 2 consecutive sets of specimens collected at least 24 hours apart from a patient with COVID-19 indicate release from quarantine. Thus far, the onset and viral shedding duration of COVID-19 are not yet known. We report here a rare case in which consecutively positive RT-PCR test results were separated by more than one month.

\section{Case Presentations}

A 37-year-old male had a history of travel from affected geographic areas (Wuhan) on January 26, 2020 and then presented symptoms of fever $\left(38.5^{\circ} \mathrm{C}\right)$, dry cough, fatigue, dizziness, runny nose and diarrhoea 5 days later. Physical examination revealed increased respiratory $(25 / \mathrm{min})$ and heart rates $(115 / \mathrm{min})$, and lung auscultation revealed a few moist rales in the right lower lobe. On admission, the patient had positive RT-PCR test results, and chest computed tomography (CT) images showed multiple nodules and mixed ground-glass opacification with consolidation in both lungs. The presence of severe acute respiratory syndrome coronavirus 2(SARS-CoV-2) in respiratory specimens was detected by real-time RT-

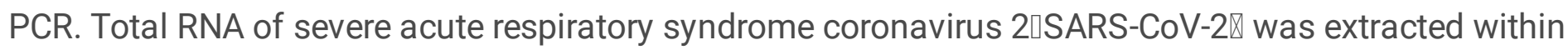
20 minutes using an RNA isolation kit (Liferiver, Wuhan, China) from throat and nasopharyngeal swab samples. Real-time reverse transcription polymerase chain reaction (RT-PCR) was performed using a SARS-CoV-2 nucleic acid detection kit according to the manufacturer's protocol (Sansure Biotech, Hunan, China). Two target genes were tested during the real-time RT-PCR assay, namely, open reading frame $1 \mathrm{ab}$ (ORF1ab) and nucleocapsid protein (N). Target 1 was ORF1ab: forward primer CCCTGTGGGTTTTACACTTAA; reverse primer ACGATTGTGCATCAGCTGA; and probe 5'-FAMCCGTCTGCGGTATGTGGAAAGGTTATGG-BHQ1-3'. Target 2 was N: forward primer GGGGAACTTCTCCTGCTAGAAT; reverse primer CAGACATTTTGCTCTCAAGCTG; and probe 5'-ROXTTGCTGCTGCTTGACAGATT-TAMRA-3'. Laboratory findings showed that the patient's lymphocyte and

$\mathrm{CD}^{+}$(cluster of differentiation 4) counts were below the normal range. Blood gas analysis and neutrophil enumeration revealed no obvious abnormities.

In this retrospectively analysed case, the hospitalized patient with COVID-19 was treated at Qian Jiang Central Hospital of ChongQing, which is a designated hospital for COVID-19 pneumonia and Public Health Clinical Center, from February 2, 2020, to March 6, 2020, and evaluated with consecutive RT-PCR tests for COVID-19 nucleic acid. The patient was diagnosed with moderate COVID-19 pneumonia 
according to the China Health Authority's interim criteria. Informed consent for the therapeutic regimen was obtained from the patient prior to treatment.

The patient received antiviral treatment, including arbidol ( $0.2 \mathrm{~g}$, tid, po), Kaletra (lopinavir $400 \mathrm{mg} /$ ritonavir $100 \mathrm{mg}, \mathrm{q} 12 \mathrm{~h}, \mathrm{po}$ ), IFN-a(interferon-a $50 \mu \mathrm{g}$, q12h, hypo), and traditional Chinese medicine, and was started on supplemental oxygen by nasal cannula after admission to the hospital. The duration of antiviral treatment was 9 days and IFN-a was 13 days. (Fig. 1). Routine blood analysis showed an increased lymphocyte count, indicating recovery and restoration of immune function. Arterial blood gas analysis showed no hypoxia. The fever, fatigue, diarrhea and rhinorrhea disappeared after four days of treatment.The cough lasted for 25 days from the illness onset. A CT scan on February 7 showed that bilateral pneumonia remained, but the appearance of the left lower lobe mildly worsened. On February 28, a chest CT scan demonstrated bilateral multiple nodules and mixed ground-glass opacification with consolidation, which were obviously improved compared with those observed on February 4, 2020 (Fig. 2). Throat and nasopharyngeal swab nucleic acid testing, COVID-19-specific antibody detection (IgG and $\operatorname{lgM}$ ), and $\mathrm{CD} 4^{+}$lymphocyte counts were evaluated regularly during the period of hospitalization (Fig. 3). The results of two continuous COVID-19 virus tests were negative for throat swabs and nasopharyngeal swabs, and the patient was thus discharged on March 6, 2020. The necleic acid test was negative from throat-swab follow up to two weeks.

\section{Discussion And Conclusions}

We report here a rare case with COVID-19, for which the longest duration of viral shedding was 32.0 days from illness onset with moderate COVID-19 pneumonia. The time from symptom onset to recovery was 36 days. Feiz et al[6] reported that the median duration of viral shedding was 20.0 days (IQR 17.0-24.0 days) in survivors, but the COVID-19 virus was detectable in patients who unfortunately died, and the longest shedding time was 37 days. That study showed that the duration of viral shedding was affected by the severity of the disease and noted that all patients in the study were hospitalized. Furthermore, prolonged viral shedding suggests that patients may still be able to transmit the new coronavirus, and prolonged shedding of the virus has important guiding significance for isolation prevention measures. Hence, non-symptomatic infected persons or recovered patients should be in in-home quarantine for an extended period of time of more than 14 days. prolonged shedding of the virus has important guiding significance for the isolation prevention measures.

Recently, many cases were positive upon RT-PCR retesting during the period of isolation and observation after discharge[7]. These findings indicated that some recovered patients may still be virus carriers.

However, Nancy Knight, an officer of the CDC's Division of Global Health Protection, said that it is possible that the oral and nasal swab positive tests detected pieces of dead virus, which would not be transmissible[8]. In the present case, consecutively positive RT-PCR tests were verified for the upper respiratory tract. The lymphocyte count from routine blood testing and $\mathrm{CD} 4^{+}$count from peripheral blood increased gradually. IgM and IgG antibodies are known to be important indicators of current, recent and past infection[9]. New coronavirus-specific IgG and IgM antibody levels changed in this case throughout 
the entire course of infection and recovery period. The recovery period of IgG antibodies is 3 times longer than that of the acute period. Therefore, the patient's upper respiratory tract swab positive test may have been caused by a piece of inactive virus.

The therapeutic procedure for this COVID-19 patient consisted of comprehensive therapy. The antiviral treatment regimen included arbidol, lopinavir/ritonavir, and interferon-a1b. The combination of therapeutic strategies is currently recommended in the latest version of China's National Health Commission-issued (version 7) official guidance. Aralen (chloroquine phosphate) is an effective antimalarial drug used to treat and prevent malaria[10]. Aralen demonstrated effectiveness against COVID-19 in a randomized multicentre controlled clinical trial (ChiCTR2000029559)[11].

In conclusion, the patient was given combination medicine treatment. All clinical symptoms and CT imaging manifestation abnormalities resolved during the course of therapy. New coronavirus-specific IgG antibody levels significantly increased by more than 3 times above those at illness onset, accompanied by decreased IgM levels. Even though the positive RT-PCR tests were verified in the upper respiratory specimens, SARS-CoV-2 may have actually changed from active to inactive, at which point it is not easily transmissible among humans and moderate COVID-19 patients. However, the shedding period of the virus is not clear in severely or extremely ill COVID-19 patients. The results warrant further independent verification in future studies; if confirmed, this information can reduce hospital bed occupancy and save medical resources.

\section{Abbreviations}

WHO:world health organization, COVID-19: coronavirus disease 2019, ARDS: severe acute respiratory11 syndrome, CDC: Center for Disease Control and Prevention, RT-PCR: real-time reverse transcriptasepolymerase chain reaction, CD4: cluster of differentiation $4, \mathrm{CT}$ : computed tomography.

\section{Declarations}

\section{Acknowledgements}

We would like to thank Zegui Li, Junyong Ding for helping to this manuscript.

\section{Authors' contributions}

$X Y L, Y H W, C Y L, Y W$ contributed to the management of this patient. CYL was the leader of the clinical team. XYL conducted the literature review and wrote the manuscript. YHW revised the article. QWM,PJPCQ PW contributed to perform isolation and identification of the pathogen and exclusion diagnosis, confirm final diagnosis. SG contributed to analyzed the radiology images. All authors read and approved the final manuscript.

\section{Funding}


This work was supported by a grant (2017ZBXM004) from Chongqing Health and

Family Planning Committee Science Foundation of China. Funding support for this project had no role in the study design, data collection and analysis, decision to publish, or preparation of this article.

\section{Availability of data and materials}

All the information supporting our conclusions and relevant references are included in the manuscript.

There are no data sets related to this case report.

\section{Ethics approval and consent to participate}

Not applicable.

\section{Consent for publication}

Written informed consent was obtained from the patient for publication of this case report.

\section{Competing interests}

The authors declare that they have no competing interests.

\section{Author details}

Departments of ${ }^{1}$ clinical laboratory, ${ }^{2}$ Neurosurgery ${ }^{4}$ radiology, ${ }^{5}$ Obstetrics, and ${ }^{6}$ Pneumology Chongqing Qiangjiang Central Hospital, the Affiliated hospital and Third Clinical College of JiShou University, No.63 Chenxi 9 Road, Qianjiang District, Chongqing 409600, P.R.China.409000. ${ }^{3}$ Qian Jiang Center for Disease Control and Prevention, No.410 xinhua east road, Qianjiang District, Chongqing 409600, P.R.China.409000. Chaoyuan Liu and Yonghong Wang contributed equally to this work, should be regarded as co-first authors.

\section{References}

[1] huang C,wang y,li x,Ren Li,Zhao J,Hu Y,Zhang L,Fan G,Xu J,Gu X,et al.Clinical features of patients infected with 2019 novel coronavirus in Wuhan,China.lancet,2020, 395 (10223), 497-506.

[2] Cui J, Li F, Shi ZL. Origin and evolution of pathogenic coronaviruses.Nat. Rev. Microbiol,2019,17,181192.

[3] World Health Organization. Novel Coronavirus(2019-nCoV) Situation Report-1,2020. Available online:https://www.who.int/docs/default-source/coronaviruse/situation-reports/20200307-sitrep-47covid-19.pdf?sfvrsn=27c364a4_4『accessed march 7,2020区.

[4] Zhu N, Zhang D, Wang W, Li X, Yang B, Song J, Zhao X, Huang B, Shi W, et al. A Novel Coronavirus from Patients with Pneumonia in China, 2019. N Engl J Med. 2020 Feb 20;382(8):727-733. doi: 
10.1056/NEJMoa2001017. Epub 2020 Jan 24.

[5] National Health Commission of the People's Republic of China. Chinese management guideline for COVID-19 (version 6.0). Feb 19, 2020. http://www.nhc.gov.cn/yzygj/s7653p

/202002/8334a8326dd94d329df351d7da8aefc2/files/b218cfeb1bc54639af227f922bf6b817.pdf (accessed Feb 19, 2020; in Chinese).

[6] Zhou F, Yu T, Du R, Fan G, Liu Y, Liu Z, Xiang J, Wang Y, Song B, Gu X, et al,Clinical course and risk factors for mortality of adult inpatients with COVID-19 in Wuhan, China: a retrospective cohort study, The Lancet,2020,https://doi.org/10.1016/S0140-6736(20)30566-3.

[7] Lan L, Xu D, Ye G, et al. Positive RT-PCR Test Results in Patients Recovered From COVID-19. JAMA. Published online February 27, 2020. doi:10.1001/jama.2020.2783.

[8]News.https://www.cbsnews.com/news/coronavirus-updates-san-antonio-woman-tests-positive-afterrelease-quarantine-may-have-exposed-dozen-others/囚accessed march 10,2020区.

[9]Li Z, Yi Y, Luo Xi, Xiong N, Liu Y, Li S, Sun R, Wang Y, Hu B, Chen W,et al. Development and Clinical Application of A Rapid IgM-IgG Combined Antibody Test for SARS-CoV-2 Infection Diagnosis. J. Med. Virol. 2020 Feb 27.doi:10.1002/jmv.25727.

[10] National Health Commission of the People's Republic of China.

http://www.nhc.gov.cn/yzygj/s7652m/202003/a31191442e29474b98bfed5579d5af95.shtml(accessed march 6, 2020; in Chinese).

[11] Cortegiani A, ngoglia G, Ippolito M, Giarratano A, Einav S. A systematic review on the efficacy and safety of chloroquine for the treatment of COVID-19,Journal of Critical Care,2020. https://doi.org/10.1016/j.jcrc.2020.03.005.

\section{Figures}




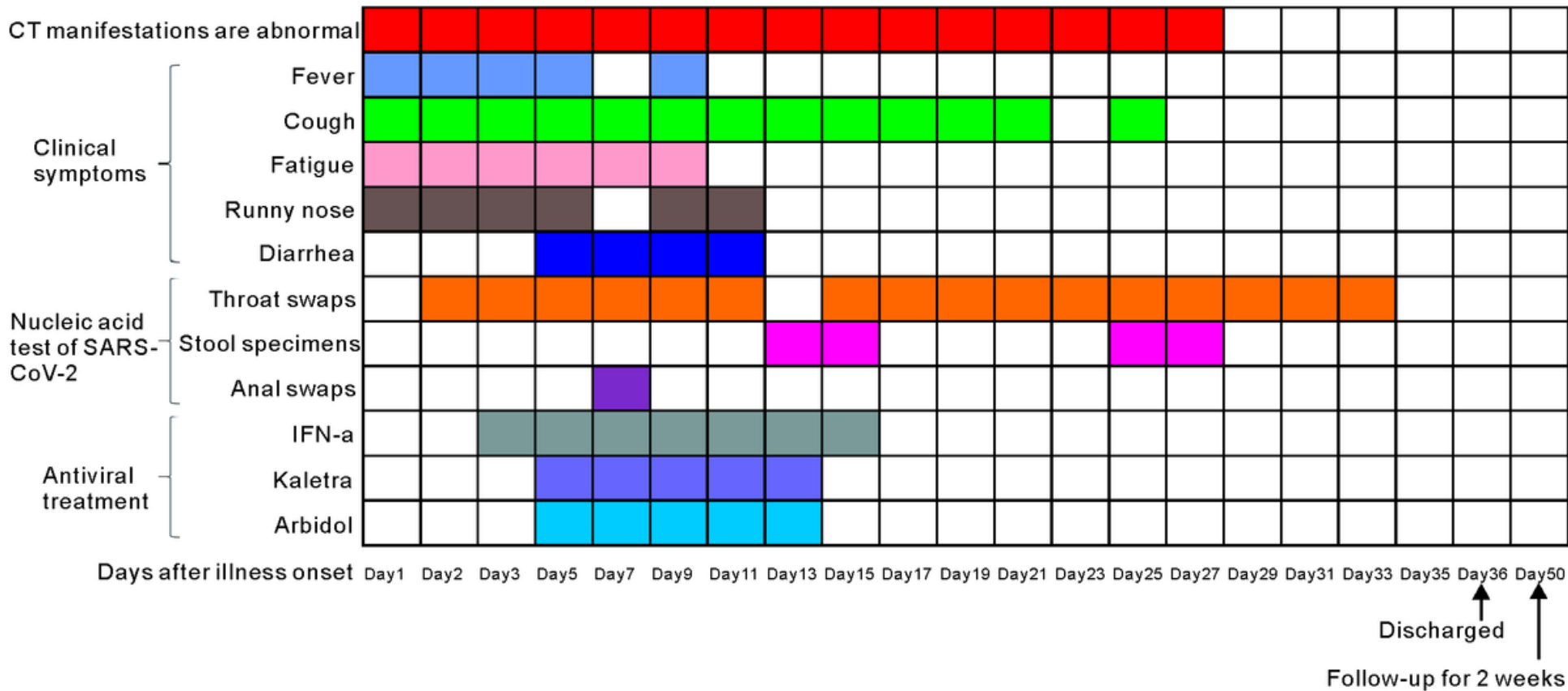

\section{Figure 1}

Clinical courses of CT scan of results,clinical symptoms,nucleic acid test of SARS-CoV-2, antiviral treatments and outcomes and duration of viral shedding from illness onset to follow up two weeks in this patient with COVID-19. (CT: computed tomography ,SARS-CoV-2囚severe acute respiratory syndrome coronavirus 2, IFNa: interferon-a,Kaletra: Iopinavir /ritonavir.) 


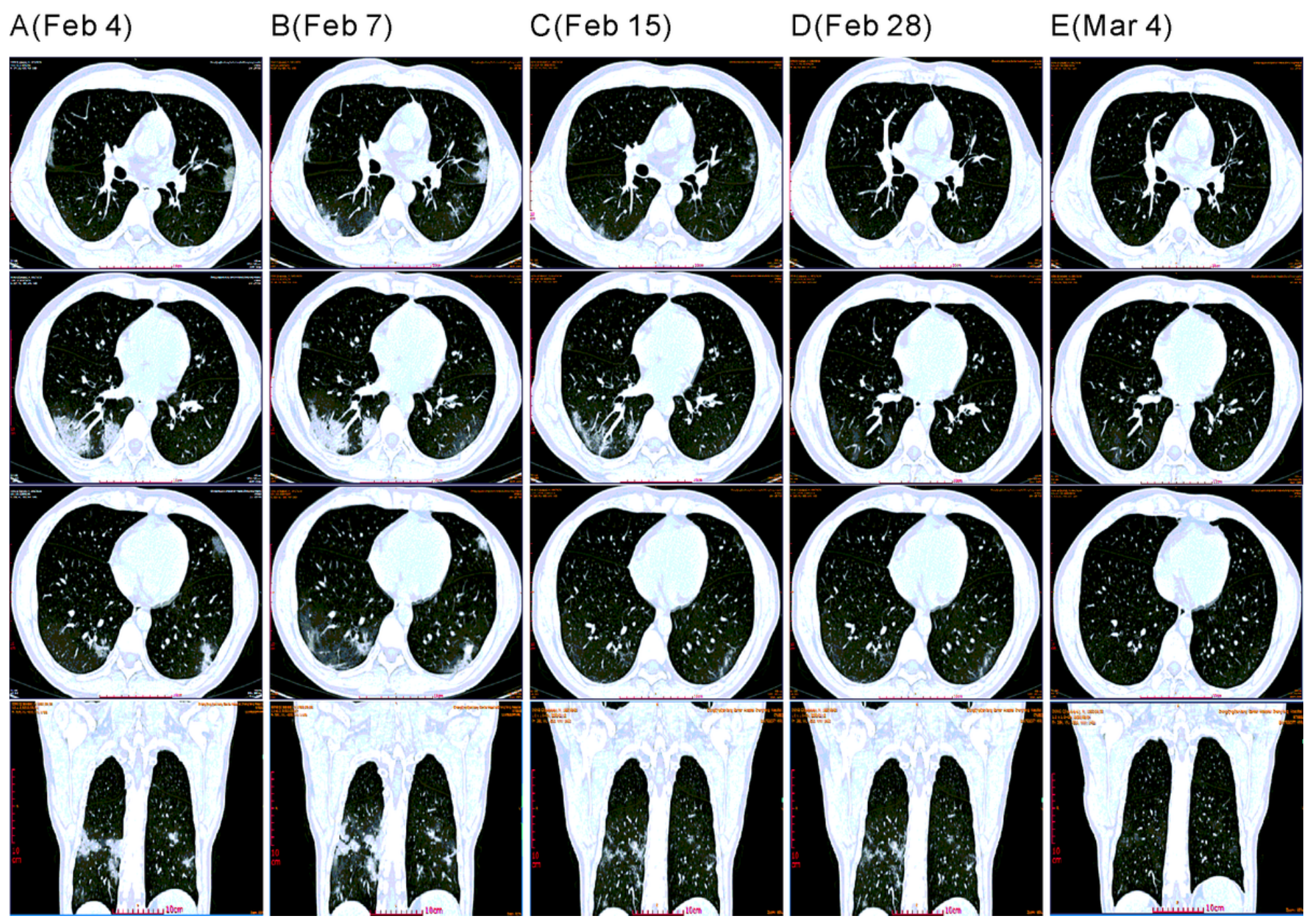

Figure 2

Chest CTs of the patient with COVID-19 obtained on February 4(A), February 7(B), February 15(C), February 28(D), February 4(E). 
A

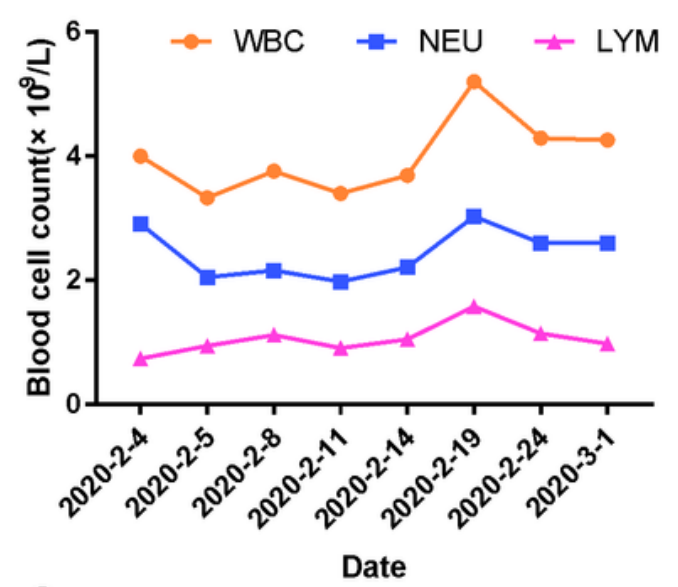

C

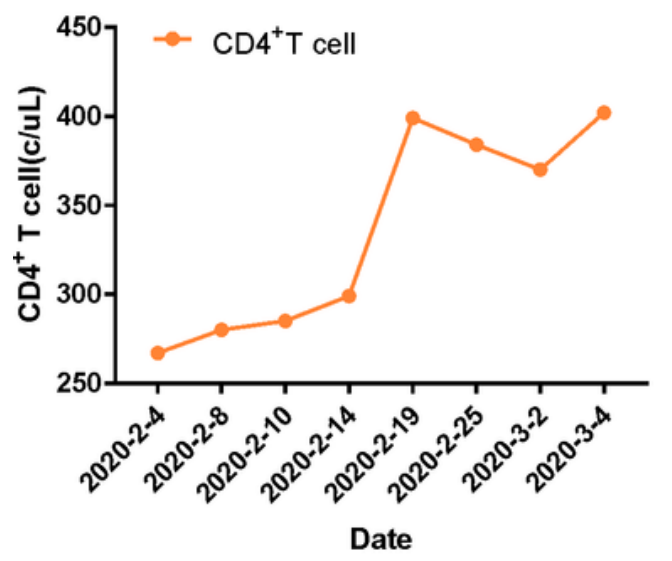

$\mathrm{E}$

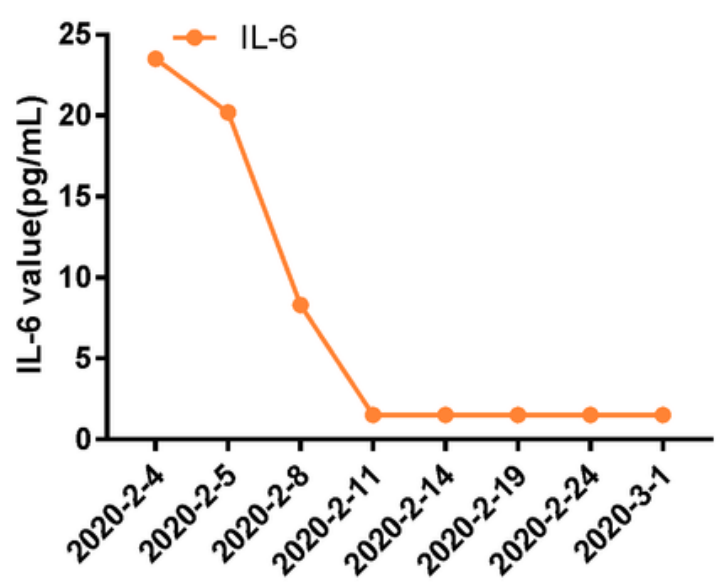

Date
B

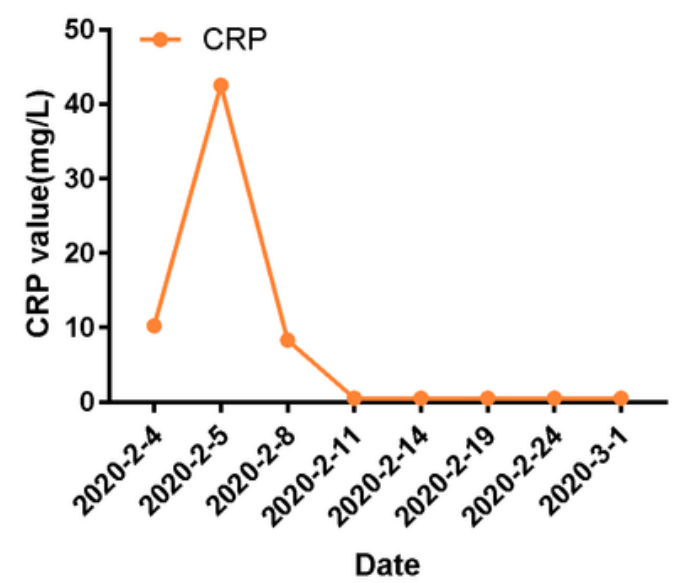

D

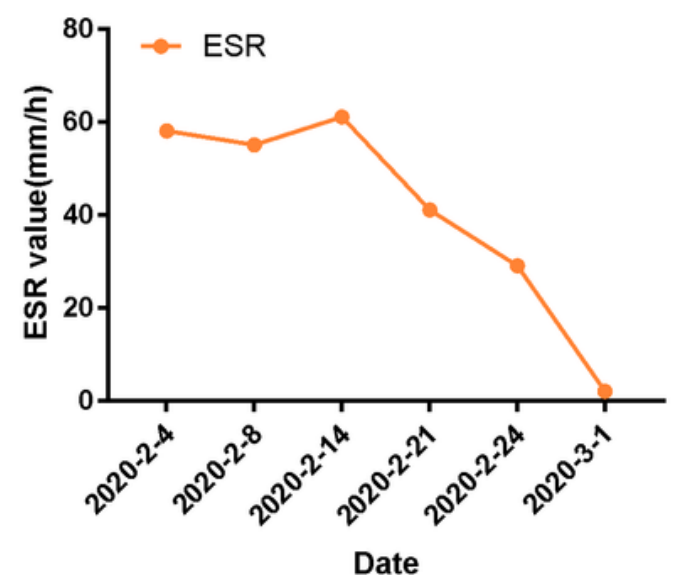

$\mathrm{F}$

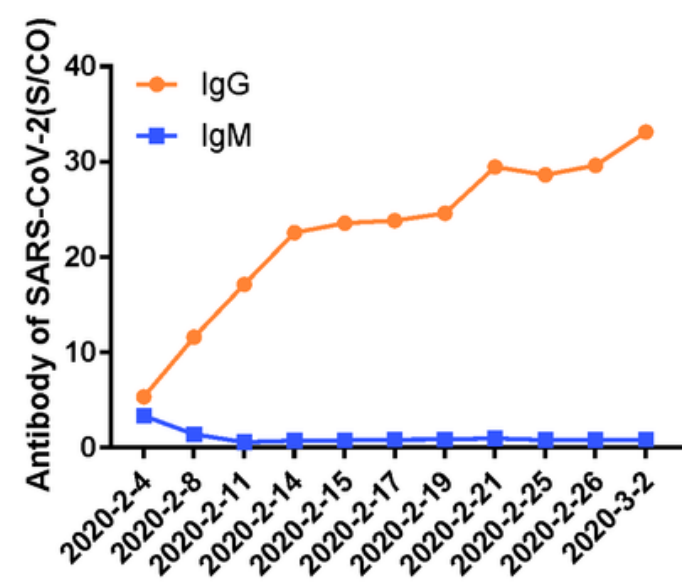

Date

Figure 3

Temporal changes in laboratory markers from illness onset in this patient hospitalised with COVID-19. Figure shows temporal changes in blood cell count(A), CRP value(B), CD4+ lymphocytes' count(C), ESR value(D), IL-6 value(E), antibody of SARS-CoV-2(F). CRP : C-Reactive Protein, CD4: Cluster of differentiation, ESR: Erythrocyte Sedimentation Rate, IL-6: Interleukin-6. WBC: White blood cell, NEU: 
Neutrophil, LYM: Lymphocyte. The increased of CRP on Februray 5 was related to the aggravation of the pneumonia.

\section{Supplementary Files}

This is a list of supplementary files associated with this preprint. Click to download.

- CAREchecklist.pdf 